\title{
Serum vitamin D levels and ovarian reserve markers in secondary amenorrhea patients: Is there a link?
}

\author{
Gyung-Mee Kim, MD, PhD', Gyun-Ho Jeon MD, PhD² \\ Departments of ${ }^{1}$ Psychiatry, ${ }^{2}$ Obstetrics and Gynecology, Inje University Haeundae Paik Hospital, Inje University College of Medicine, Busan, Korea
}

\section{Objective}

To investigate whether serum 25-hydroxyvitamin $D[25(\mathrm{OH}) \mathrm{D}]$ level is associated with ovarian reserve markers in secondary amenorrhea (SA) patients.

\section{Methods}

Sixty-three women diagnosed with SA were recruited during 12 months from the initiation of this prospective observational study. Serum 25(OH)D levels, serum anti-Müllerian hormone (AMH) levels and antral follicle count (AFC) were estimated in study participants and ovarian reserve markers were compared between participants with vitamin $D$ deficiency and those with normal vitamin D levels.

Results

Of the 63 participants, $27(42.9 \%)$ were vitamin D deficient $(<20 \mathrm{ng} / \mathrm{mL})$ and $36(57.1 \%)$ had normal vitamin D levels. The mean AMH levels and AFC were 10.86 $\pm 8.94 \mu / L$ and $15.23 \pm 7.65$ in the vitamin D deficient group, and 7.24 $\pm 5.62 \mu / L$ and $12.30 \pm 6.95$ in the normal vitamin $D$ group. Univariate and multivariate linear regression analysis of $\log _{10}$ transformed AMH and AFC with serum 25(OH)D adjusted for age and body mass index confirmed no association between vitamin D levels and AMH levels or AFC. There was also no correlation between serum 25(OH)D and AMH levels or AFC in all participants. However, participants with vitamin D deficiency had an increased chance of having polycystic ovarian syndrome (PCOS) as cause of SA than those with normal vitamin D levels (adjusted odds ratio, 7.559; $95 \%$ confidence interval, 1.28-44.65; $P=0.026$ ) after adjustment for clinical factors by logistic regression model.

\section{Conclusion}

There was no correlation between serum 25(OH)D levels and ovarian reserve markers in SA patients, but vitamin D deficiency may be linked to PCOS patients.

Keywords: Anti-Mullerian hormone; Amenorrhea; Ovarian reserve; 25-hydroxyvitamin D; Polycystic ovarian syndrome

\section{Introduction}

Vitamin $D$ is known to have a major influence on bone health through regulation of calcium phosphorus homeostasis. Recently, the female reproductive system has been defined as one of the non-classical target organs of vitamin $D$. Vitamin $D$ receptor expression was identified in ovarian granulosa cells as well as other female reproductive organs, including endometrium and the uterus. These findings suggest that vitamin D may have a potential role in female reproduction [1-3]. Several studies have demonstrated a direct effect of vitamin D on ovarian folliculogenesis and steroidogenesis in animal and human cell-line studies; vitamin D receptor null mutant mice have impaired folliculogenesis and vitamin $D$
Received: 2020.03.19. Revised: 2020.05.13. Accepted: 2020.06.01. Corresponding author: Gyun-Ho Jeon, MD, PhD

Department of Obstetrics and Gynecology, Inje University Haeundae Paik Hospital, Inje University College of Medicine, 875 Haeun-daero, Haeundae-gu,Busan 48108, Korea

E-mail: jeon285@hotmail.com

https://orcid.org/0000-0003-1128-1656

Articles published in Obstet Gynecol Sci are open-access, distributed under the terms of the Creative Commons Attribution Non-Commercial License (http://creativecommons. org/licenses/by-nc/3.0/) which permits unrestricted non-commercial use, distribution, and reproduction in any medium, provided the original work is properly cited.

Copyright $\odot 2020$ Korean Society of Obstetrics and Gynecology 


\section{Obstetrics \& Gynecology Science}

Vol. 63, No. 4, 2020

stimulated steroidogenesis in human ovarian cells $[4,5]$.

Anti-Müllerian hormone (AMH) is produced by granulosa cells of small antral and pre-antral follicles, and along with antral follicle counts (AFCs), is a well-known representative marker for ovarian reserve. AMH plays a crucial role in folliculogenesis and is not affected by the menstrual cycle, making it the most widely used ovarian marker. Although it is still unclear how vitamin $D$ affects female reproductive function, interest in a potential relationship between vitamin $\mathrm{D}$ and ovarian reserve markers, particularly $\mathrm{AMH}$, has increased since reports on the effect of vitamin $D$ on follicular development have accumulated. Moreover, the presence of a functional vitamin $\mathrm{D}$ response element in the $\mathrm{AMH}$ gene promotor region was demonstrated [6] and experimental results in animal and human granulosa cells have also reported that vitamin D affects AMH signaling $[7,8]$. These basic research studies have indicated that vitamin D deficiency may alter gonadal function through abnormal AMH signaling. However, discrepancy exists in clinical study findings under various settings, where some suggest a significant association between vitamin $\mathrm{D}$ levels and ovarian reserve, while others have not found any correlation between vitamin $D$ and ovarian reserve markers $[9,10]$. Considering these contradictory results, we performed this prospective observational study to examine serum 25-hydroxyvitamin D [25(OH)D] levels in patients with secondary amenorrhea $(S A)$ and investigate the relationship between serum $25(\mathrm{OH}) \mathrm{D}$ levels and ovarian reserve markers (i.e., serum $\mathrm{AMH}$ and $\mathrm{AFC}$ ) in these women.

\section{Materials and methods}

\section{Study design and participants}

This study was conducted as a prospective cohort study for 12 months from March 2018 to February 2019. The study population was comprised of patients evaluated by a single reproductive endocrinologist for symptoms of SA. A total of 78 participants were initially recruited to the study. Three participants refused to participate in the study and 12 participants were excluded due to the study's exclusion criteria. The exclusion criteria of this study were as follows: 1) women who had taken hormonal medication including oral contraceptives or vitamin D supplements within the previous 6 months; 2) women who had ovarian surgery, chemotherapy or radiotherapy; 3) women who took medication and/or were diag- nosed with a systemic disease that can affect menstruation (e.g., psychoaffective medicine, thyroid hormone, diabetes, hyperprolactinemia); 4) women who refused to participate this study. Patients' information including age, parity, height, body weight, cause of SA, season of blood sampling (i.e., to account for seasonal changes in vitamin D), current medications and other past medical history were recorded. Gynecologic ultrasonography was also performed on their first study visit. To determine cause of SA, polycystic ovarian syndrome (PCOS) was diagnosed on the basis of Rotterdam criteria [11], and primary ovarian insufficiency (POI) was regarded as 2 recordings of serum follicular stimulating hormone (FSH) levels of more than $40 \mathrm{IU} / \mathrm{L}$ at least one month apart in a woman aged under 40 years (i.e., >2 standard deviation [SD] less than the mean menopausal age) [12]. All patients whose etiology of SA was not determined, excluding women with $\mathrm{POI}$ and PCOS, were deemed unexplained chronic anovulation [13]. PCOS in adolescents was only diagnosed in women with at least 2 years after menarche and who had SA, hyperandrogenemia, and increased ovarian volume on ultrasound [14].

Venous blood samples of all participants were taken for the measurement of serum $25(\mathrm{OH}) \mathrm{D}$ and $\mathrm{AMH}$ on the same day, prior to treatment for SA. Vitamin D deficiency was defined as serum 25(OH)D levels $<20 \mathrm{ng} / \mathrm{mL}$ based on the Endocrine Society clinical practice guidelines [15].

\section{Serum vitamin $D$, hormones and ovarian reserve markers measurements}

\section{1) Serum 25-hydroxyvitamin $D$}

Each participant's venous sample was drawn into a serum separation tube (SST) and serum 25(OH)D was measured using a DIA source $25 \mathrm{OH}$ Vitamin D3 radioimmunoassay kit (DIAsource ImmunoAssays S.A., Louvain-La-Neuve, Belgium) with immunoassay device (gamma 5) and presented in $\mathrm{ng} / \mathrm{mL}$. The total imprecision coefficient of variance was $5.38 \%$ at a concentration level of $6.35 \mathrm{ng} / \mathrm{mL}$ and $4.96 \%$ at $37.71 \mathrm{ng} / \mathrm{mL}$.

\section{2) Serum follicular stimulating hormone}

Each participant's venous sample was drawn into an SST and serum FSH was measured using an Elecsys FSH electrochemiluminescence immunoassay kit (Roche Diagnostics $\mathrm{GmbH}$, Mannheim, Germany) with immunoassay device (Cobas e 801 ) and presented in $\mathrm{mIU} / \mathrm{mL}$. The total imprecision coef- 


\section{Obstetrics \& Gynecology Science}

Gyung-Mee Kim, et al. Vitamin D and ovarian reserve markers

ficient of variance was $3.1 \%$ at a concentration level of $48.8 \mathrm{mlU} / \mathrm{mL}$ and $2.3 \%$ at $17.4 \mathrm{mlU} / \mathrm{mL}$.

3) Anti-Müllerian hormone and antral follicle counts Blood drawn into the SST was centrifuged within 1 hour (3,000 rpm for 10 minutes). The separated serum was measured for AMH level by ECLIA method with Elecsys AMH kit (Roche Diagnostics $\mathrm{GmbH}$ ) on a Cobas e 601 immunoassay analyzer and presented in $\mathrm{ng} / \mathrm{mL}$. The total imprecision coefficient of variance was $3.5 \%$ at a concentration level of $0.042 \mathrm{ng} / \mathrm{mL}$ and $3.4 \%$ at $0.20 \mathrm{ng} / \mathrm{mL}$. An AFC was measured by one gynecologist on the participant's first visit in all individuals with gynecologic ultrasonography. AFC represented the total number of all antral follicles, from $2 \mathrm{~mm}$ to $10 \mathrm{~mm}$ in size, in both ovaries.

\section{Statistical analysis}

Categorical variable data are presented as a frequency with percentage and continuous variables are presented as a group mean $\pm S D$. Differences in study participants' characteristics were compared across subgroups with a $\chi^{2}$ test or
Fisher's exact test for categorical variables and independent t-test or Mann-Whitney's $U$ test for continuous variables, as appropriate. An analysis of variance with Duncan's post hoc test or Kruskal-Wallis test with Dunn's post hoc test were also employed, as appropriate. To check if data were normally distributed, we used a Shapiro-Wilk test. Partial correlation coefficients controlling for age and body mass index (BMI) were estimated to investigate the linear relationship between 2 continuous variables. Univariate linear regression analysis between each variable (age, BMl, vitamin $\mathrm{D}$, season), and multivariate linear regression analysis with all covariates, were performed to identify the regression coefficients for factors related to the $\log _{10}$ AMH, AFC, PCOS and POI. For data visualization, box plot and scatter plot were also displayed. Finally, logistic regression analysis was performed to identify whether vitamin D deficiency is associated with PCOS as a cause of SA. All statistical analyses were carried out using SPSS 24.0 (SPSS Statistics for Windows 24.0; IBM Corp., Armonk, NY, USA) statistical software and P-values less than 0.05 was considered statistically significant.

Table 1. Patients' demographic variables

\begin{tabular}{|c|c|c|c|}
\hline \multirow{2}{*}{ Variable } & \multicolumn{2}{|c|}{ Vitamin D (ng/mL) } & \multirow{2}{*}{$P$-value } \\
\hline & $<20$ & $\geq 20$ & \\
\hline Age (yr) & $22.54 \pm 5.33$ & $27.81 \pm 8.65$ & $0.014^{a)}$ \\
\hline $\mathrm{BMI}\left(\mathrm{kg} / \mathrm{m}^{2}\right)$ & $22.85 \pm 5.17$ & $22.56 \pm 4.39$ & $0.823^{\mathrm{a})}$ \\
\hline \multicolumn{4}{|l|}{ Amenorrhea cause } \\
\hline PCOS & $21(80.8)$ & $19(51.4)$ & $0.454^{b)}$ \\
\hline Unexplained chronic anovulation & $2(7.7)$ & $12(32.4)$ & \\
\hline $\mathrm{POI}$ & $3(11.5)$ & $6(16.2)$ & \\
\hline $\mathrm{AFC}$ & $15.23 \pm 7.65$ & $12.30 \pm 6.95$ & $0.119^{c)}$ \\
\hline $\mathrm{AMH}$ & $10.86 \pm 8.94$ & $7.24 \pm 5.62$ & $0.130^{a)}$ \\
\hline FSH & $6.30 \pm 4.28$ & $8.25 \pm 9.39$ & $0.392^{a)}$ \\
\hline Season of blood sample & & & $0.128^{b)}$ \\
\hline Autumn & $6(23.1)$ & $2(5.4)$ & \\
\hline Spring & $9(34.6)$ & $11(29.7)$ & \\
\hline Summer & $6(23.1)$ & $16(43.2)$ & \\
\hline Winter & $5(19.2)$ & $8(21.6)$ & \\
\hline
\end{tabular}

Values are presented as mean \pm standard deviation or number (\%).

BMI, body mass index; PCOS, polycystic ovarian syndrome; POI, primary ovarian insufficiency; AFC, antral follicle count; AMH, anti-Müllerian hormone; FSH, follicular stimulating hormone.

${ }^{\text {a) }} P$-values were derived from Mann-Whitney $U$ test; ${ }^{b} P$-values were derived from Fisher's exact test; ${ }^{c} P$-values were derived from independent $t$ test. 


\title{
Obstetrics \& Gynecology Science
}

\author{
Vol. 63 , No. 4, 2020
}

\section{Results}

Sixty-three participants with SA were finally included in this study. The mean age of participants was $26.11 \pm 8.05$ years and the mean BMI was $22.75 \pm 4.75 \mathrm{~kg} / \mathrm{m}^{2}$. Participants' baseline and biochemical characteristics of patients are shown in Table 1. Of all participants, $42.9 \%(n=27)$ were vitamin D deficient $(<20 \mathrm{ng} / \mathrm{mL})$ and $57.1 \%(\mathrm{n}=36)$ had normal vitamin $D$ levels ( $\geq 20 \mathrm{ng} / \mathrm{mL}$ ). The mean AMH levels and AFC were $10.86 \pm 8.94 \mu / L$ and $15.23 \pm 7.65$ in the vitamin $D$ deficient group, and 7.24 $\pm 5.62 \mu / L$ and $12.30 \pm 6.95$ in the normal vitamin $\mathrm{D}$ group, respectively. The difference in $\mathrm{BMI}$, cause of amenorrhea, season of blood sample and FSH levels between the 2 groups did not reach statistical significance, but age was significantly higher in the normal vitamin $D$ group.

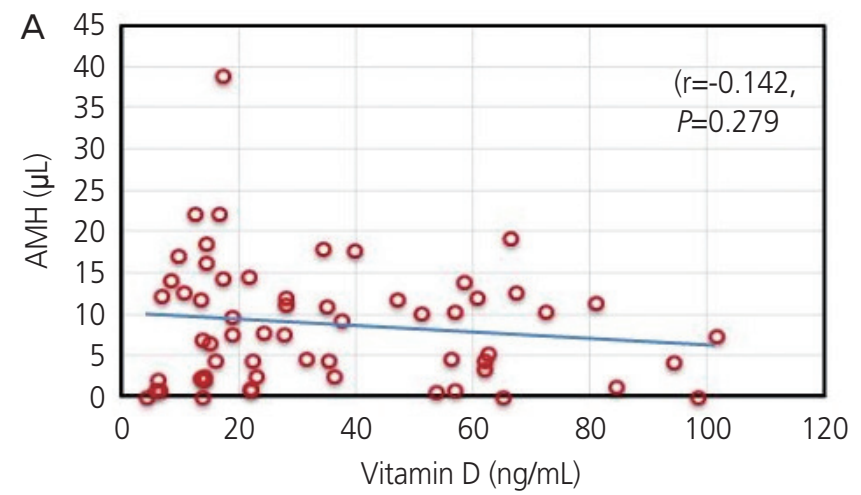

There was no correlation between serum vitamin $D$ and AMH levels $(r=-0.142, P=0.279)$ or AFC $(r=-0.101, P=0.433)$ in all participants (Fig. 1). After univariate linear regression analysis of $\log _{10}$ transformed AMH or AFC with all covariates, multivariate linear regression analysis of $\log _{10}$ transformed $\mathrm{AMH}$ or AFC with vitamin $\mathrm{D}$, age and BMI was performed with adjustment for confounding variables. While there was a significant association between serum AMH levels and age, there were no significant relationships between serum $\mathrm{AMH}$ levels or AFC and serum vitamin D levels: regression coefficient for vitamin $D$ predicting $\log _{10} \mathrm{AMH}$ : -0.002 , standard error (SE): 0.004, $P=0.557$ (Table 2), regression coefficient for vitamin D predicting AFC: -0.042, SE: $0.040, P=0.304$ (Table 3). In the univariate and multivariate linear regression of PCOS modelled with vitamin D levels, there was also no significant association between PCOS and serum vitamin D

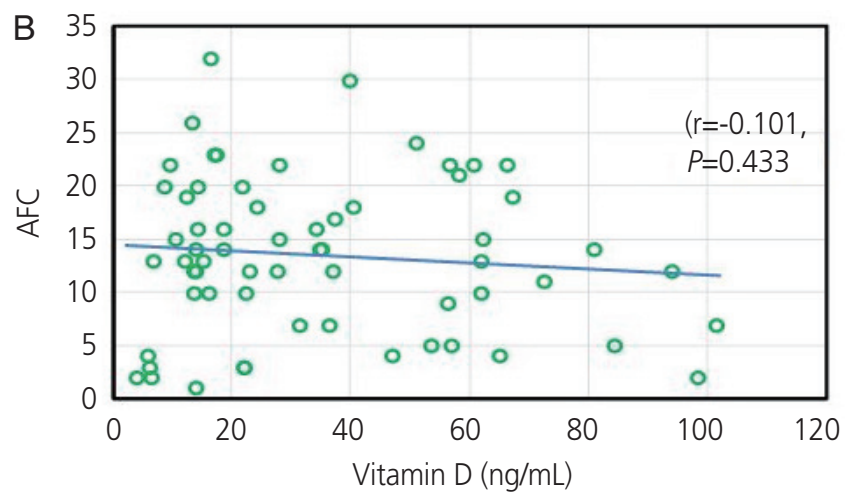

Fig. 1. No correlation between serum vitamin $D$ and anti-Müllerian hormone (AMH) levels $(A)$ or antral follicle counts (AFCs) (B).

Table 2. Regression coefficients in univariate and multivariate linear regression analysis between all covariates and serum anti-Müllerian hormone $(\mathrm{AMH})$

\begin{tabular}{|c|c|c|c|c|c|c|}
\hline \multirow{2}{*}{ Covariates } & \multicolumn{3}{|c|}{ Univariate } & \multicolumn{3}{|c|}{ Multivariate } \\
\hline & Estimate \pm SE & $\mathbf{t}$ & $P$-value & Estimate $\pm \mathrm{SE}$ & $\mathbf{t}$ & $P$-value \\
\hline Vitamin D & $-0.007 \pm 0.004$ & -1.962 & 0.055 & $-0.002 \pm 0.004$ & -0.591 & 0.557 \\
\hline Age (yr) & $-0.031 \pm 0.011$ & -2.867 & 0.006 & $-0.034 \pm 0.013$ & -2.541 & 0.014 \\
\hline BMI $\left(\mathrm{kg} / \mathrm{m}^{2}\right)$ & $0.008 \pm 0.021$ & 0.370 & 0.713 & $0.020 \pm 0.022$ & 0.937 & 0.353 \\
\hline \multicolumn{7}{|c|}{ Season of blood sample } \\
\hline Winter & - & - & & & & \\
\hline Spring & $-0.177 \pm 0.247$ & -0.715 & 0.478 & & & \\
\hline Summer & $0.301 \pm 0.237$ & 1.272 & 0.208 & & & \\
\hline Autumn & $-0.310 \pm 0.309$ & -1.002 & 0.320 & & & \\
\hline
\end{tabular}

Dependent variable: $\log _{10} \mathrm{AMH}$.

$\mathrm{SE}$, standard error; BMI, body mass index. 


\section{Obstetrics \& Gynecology Science}

Gyung-Mee Kim, et al. Vitamin D and ovarian reserve markers

levels (Table 4). The results of univariate and multivariate linear regression of $\mathrm{POI}$ modelled with vitamin $\mathrm{D}$ levels was also similar: regression coefficient \pm SE: $0.001 \pm 0.002, P=0.641$, $-0.001 \pm 0.002, P=0.557$, respectively). However, serum vitamin D levels for each SA cause group demonstrated lower tendency for vitamin D levels in the PCOS or POI groups than in the unexplained chronic anovulation group (31.07 \pm 22.05 , $31.37 \pm 31.89,48.92 \pm 27.69$, respectively, $P=0.056$ ) (Fig. 2). In the logistic regression analysis, women with vitamin $D$ deficiency had an increased chance of having PCOS as cause of SA than those with normal vitamin D levels (adjusted odds ratio $[O R], 7.559 ; 95 \%$ confidence interval, 1.28-44.65; $P=0.026)$ after adjusting for $\mathrm{BMI}$, age and seasonal variation of vitamin $\mathrm{D}$.

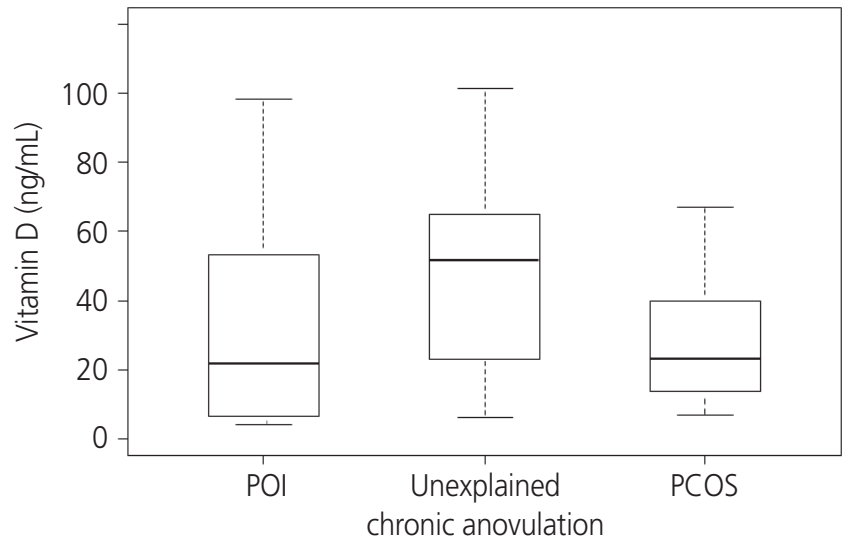

Fig. 2. Comparison of serum vitamin $D$ levels among primary ovarian insufficiency (POI) group, unexplained chronic anovulation group and polycystic ovarian syndrome (PCOS) group.

Table 3. Regression coefficients in univariate and multivariate linear regression analysis between all covariates and antral follicle counts (AFCs)

\begin{tabular}{|c|c|c|c|c|c|c|}
\hline \multirow{2}{*}{ Covariates } & \multicolumn{3}{|c|}{ Univariate } & \multicolumn{3}{|c|}{ Multivariate } \\
\hline & Estimate $\pm S E$ & $t$ & $P$-value & Estimate $\pm S E$ & $t$ & $P$-value \\
\hline Vitamin D & $-0.066 \pm 0.036$ & -1.836 & 0.071 & $-0.042 \pm 0.040$ & -1.038 & 0.304 \\
\hline Age (yr) & $-0.188 \pm 0.110$ & -1.720 & 0.090 & $-0.178 \pm 0.137$ & -1.294 & 0.201 \\
\hline BMI $\left(\mathrm{kg} / \mathrm{m}^{2}\right)$ & $0.121 \pm 0.189$ & 0.636 & 0.527 & $0.191 \pm 0.207$ & 0.920 & 0.361 \\
\hline \multicolumn{7}{|c|}{ Season of blood sample } \\
\hline \multicolumn{7}{|c|}{ Winter } \\
\hline Spring & $1.400 \pm 2.394$ & 0.585 & 0.561 & & & \\
\hline Summer & $4.374 \pm 2.327$ & 1.880 & 0.065 & & & \\
\hline Autumn & $-2.300 \pm 3.069$ & -0.749 & 0.456 & & & \\
\hline
\end{tabular}

Dependent variable: AFCs.

$\mathrm{SE}$, standard error; BMI, body mass index.

Table 4. Regression coefficients in univariate and multivariate linear regression analysis between all covariates and polycystic ovarian syndrome (PCOS)

\begin{tabular}{|c|c|c|c|c|c|c|}
\hline \multirow{2}{*}{ Covariates } & \multicolumn{3}{|c|}{ Univariate } & \multicolumn{3}{|c|}{ Multivariate } \\
\hline & Estimate $\pm S E$ & $\mathbf{t}$ & $P$-value & Estimate $\pm S E$ & $\mathbf{t}$ & $P$-value \\
\hline Vitamin D & $-0.006 \pm 0.002$ & -2.521 & 0.014 & $-0.005 \pm 0.003$ & -1.815 & 0.075 \\
\hline Age (yr) & $-0.011 \pm 0.007$ & -1.425 & 0.159 & $-0.008 \pm 0.009$ & -0.916 & 0.363 \\
\hline $\mathrm{BMI}\left(\mathrm{kg} / \mathrm{m}^{2}\right)$ & $0.015 \pm 0.013$ & 1.158 & 0.251 & $0.017 \pm 0.013$ & 1.228 & 0.224 \\
\hline \multicolumn{7}{|c|}{ Season of blood sample } \\
\hline Winter & - & & & & & \\
\hline Spring & $0.067 \pm 0.165$ & 0.404 & 0.687 & & & \\
\hline Summer & $0.249 \pm 0.160$ & 1.556 & 0.125 & & & \\
\hline Autumn & $-0.033 \pm 0.211$ & -0.158 & 0.875 & & & \\
\hline
\end{tabular}

Dependent variable: PCOS.

$\mathrm{SE}$, standard error; BMI, body mass index. 


\title{
Obstetrics \& Gynecology Science
}

\author{
Vol. 63, No. 4, 2020
}

\section{Discussion}

Our study demonstrated that serum 25(OH)D levels were not related with ovarian reserve markers among SA patients, even after adjustment for age, BMI, cause of amenorrhea, and seasonal variation of vitamin D. However, mean serum vitamin $D$ levels were different depending on the cause of amenorrhea, however the difference did not reach statistical significance (Fig. 2). In particular, vitamin D levels in the participants with PCOS or POI demonstrated a tendency towards lower serum vitamin $D$ levels than those in participants with unexplained chronic anovulation. In addition, women with vitamin D deficiency had an increased chance of having PCOS than those with normal vitamin D levels, after adjustment for clinical factors by logistic regression model. We did not calculate the adjusted OR for POI since participants with POI were a very small portion of the study participants. The lack of correlation between serum vitamin $D$ levels and ovarian reserve markers across all participants in our study is in agreement with several recent studies that also demonstrated no correlation. However, the result of lower vitamin $D$ levels in participants with PCOS in this study needs to be taken with caution, considering the heterogeneous results of previous studies $[16,17]$. To date, the role of low vitamin $D$ levels on $\mathrm{AMH}$ regulation or the pathophysiology of PCOS is unclear, with some previous reports suggesting that this vitamin $D$ deficiency is likely due to obesity or a metabolic syndrome that is frequently associated with PCOS $[18,19]$.

While basic research has reported that vitamin $D$ affects ovarian folliculogenesis and steroidogenesis, there is limited clinical information on how vitamin D affects ovarian markers such as AMH. Wojtusik et al. [9] have reported a dosedependent decrease in AMH mRNA levels in granulosa cells after treatment with vitamin D. In another recent study, human granulosa cells treated with vitamin $D$ exhibited altered AMH signaling and they demonstrated an inverse correlation between vitamin D status in follicular fluid and AMH receptor-II (AMHT-II) mRNA gene expression [8]. Binding AMH to AMH receptor-II (AMHR-II) is known to suppress follicular maturation, by inhibiting primordial follicle recruitment into the growing follicle pool, and by decreasing the sensitivity of follicles to FSH [10]. Since vitamin D had an effect on downregulation of AMHR-\|l gene expression, the phosphorylation process, and nuclear localization after AMH-AMHR-II binding [8], it may be involved in promoting follicle development by altering $\mathrm{AMH}$ production pattern and FSH sensitivity in ovarian granulosa cell $[20,21]$.

Previous studies on the relationship between vitamin $D$ and ovarian reserve markers, particularly $\mathrm{AMH}$, have had inconsistent results. For instance, Merhi et al. [22] found a positive relationship between serum vitamin $D$ and $A M H$ levels and suggested vitamin $D$ deficiency might be associated with lower ovarian reserve in late reproductive aged women. Dennis et al. [23] also suggested that vitamin D might have a positive effect on $\mathrm{AMH}$ production in adults, and the extent of seasonal variation in women's AMH levels correlated with the extent of their variation in vitamin $D$ levels. In another study of 1,430 premenopausal women, a negative correlation was found between serum vitamin $D$ levels and urinary FSH levels, suggesting that low vitamin D levels may influence ovarian reserve and earlier menopause [24]. Meanwhile, a recent cross-sectional study that included 283 infertile women, revealed no significant association between vitamin $D$ and ovarian reserve markers (AMH, AFC) [25], which is in agreement with a prospective study that failed to demonstrate any beneficial effect of vitamin $D$ supplementation on normalization of serum AMH levels [26].

In this study, generally healthy women should be included as controls. However, it was difficult to recruit healthy women to volunteer to have their $\mathrm{AMH}$ and vitamin $\mathrm{D}$ levels measured, so we conducted this study in patients with SA who required $\mathrm{AMH}$ measurements for diagnosis. These SA patients included women with various levels of ovarian reserve, and it was assumed that unexplained chronic anovulatory patients were similar to healthy women with normal ovarian reserve. Indeed, participants with unexplained chronic anovulatory in our study demonstrated normal ovarian reserve (data not shown). In addition, cholecalciferol, the main natural source of vitamin D, is affected by sun exposure and outdoor activity, so participants' duration of outdoor activity should also be measured. However, we did not conduct a survey of patients' outdoor activity as subjective factors could have been reflected in this evaluation. Although the validity of AMH measurements in those younger than 20 years of age has not been established, we included participants in this age group and their AMH measurement, as the small number of girls in their late teens in this study were considered to have reached sufficient maturity.

This study is the first report on the relationship between serum vitamin $\mathrm{D}$ levels and ovarian reserve in Korean women. 


\section{Obstetrics \& Gynecology Science}

Gyung-Mee Kim, et al. Vitamin D and ovarian reserve markers

This study was possible because it was conducted in patients with SA, which included women with various levels of ovarian reserves. Furthermore, this study is strengthened by its prospective design, which has a low risk of confounding bias, the fact that both AFC and AMH were measured as a marker of ovarian reserve, and finally $\mathrm{AMH}$ was measured on the same day as $25(\mathrm{OH}) \mathrm{D}$.

However, this study has several limitations. Firstly, this study has small study population to then analyze by subgroup(s) of amenorrhea causes, which increases the risk of selection bias or type II error. Secondly, healthy women with normal menstrual cycles, who were not SA patients, were not included as controls. Thirdly, this study was observational in nature, so we cannot interpret these results in terms of causality. Despite of these limitations, this study is the first prospective observational study of the relationship between vitamin $D$ and ovarian reserve in Korean women with SA, suggesting no association between them. In this regard, it is not necessary to routinely measure serum vitamin $D$ levels in SA patients with abnormal ovarian reserve. However, it would be meaningful to provide vitamin D supplementation, after measuring serum vitamin D concentration in PCOS patients, considering the low vitamin D levels in PCOS patients in this study. Nevertheless, given the uncertainty of how vitamin D affects female reproduction and the limitations of this study, no clear conclusion can be drawn. Further study is required on the relationship between vitamin D levels and obesity or metabolic abnormalities in patients with PCOS.

In conclusion, this observational study demonstrated that there was no correlation between serum vitamin D levels and ovarian reserve markers in SA patients, but that vitamin D deficiency may be linked to PCOS patients. However, given the limitations of this observational study, further prospective research with a larger population and age-matched control group should be investigated to draw more definite conclusions on the relationship between vitamin $\mathrm{D}$ levels and ovarian reserve markers.

\section{Acknowledgements}

This work was supported by the 2018 Inje University research grant (grant number 20180150).

\section{Conflict of interest}

No potential conflict of interest relevant to this article was reported.

\section{Ethical approval}

This study was approved by the Institutional Review Board of Inje University Haeundae Paik Hospital, Busan, Korea (approval number: 2017-01-018).

\section{Patient consent}

All participants provided written informed consent.

\section{References}

1. Fabris A, Pacheco A, Cruz M, Puente JM, Fatemi H, Garcia-Velasco JA. Impact of circulating levels of total and bioavailable serum vitamin $D$ on pregnancy rate in egg donation recipients. Fertil Steril 2014;102:1608-12.

2. van de Vijver A, Drakopoulos P, Van Landuyt L, Vaiarelli A, Blockeel C, Santos-Ribeiro $S$, et al. Vitamin D deficiency and pregnancy rates following frozen-thawed embryo transfer: a prospective cohort study. Hum Reprod 2016;31:1749-54.

3. Franasiak JM, Molinaro TA, Dubell EK, Scott KL, Ruiz AR, Forman EJ, et al. Vitamin D levels do not affect IVF outcomes following the transfer of euploid blastocysts. Am J Obstet Gynecol 2015;212:315.e1-6.

4. Yoshizawa T, Handa Y, Uematsu Y, Takeda S, Sekine K, Yoshihara $Y$, et al. Mice lacking the vitamin $D$ receptor exhibit impaired bone formation, uterine hypoplasia and growth retardation after weaning. Nat Genet 1997;16:391-6.

5. Parikh G, Varadinova M, Suwandhi P, Araki T, Rosenwaks $Z$, Poretsky $L$, et al. Vitamin D regulates steroidogenesis and insulin-like growth factor binding protein-1 (IGFBP-1) production in human ovarian cells. Horm Metab Res 2010;42:754-7.

6. Malloy PJ, Peng L, Wang J, Feldman D. Interaction of the vitamin $D$ receptor with a vitamin $D$ response element in 


\section{Obstetrics \& Gynecology Science}

Vol. 63, No. 4, 2020

the Mullerian-inhibiting substance (MIS) promoter: regulation of MIS expression by calcitriol in prostate cancer cells. Endocrinology 2009;150:1580-7.

7. Dicken $C L$, Israel DD, Davis JB, Sun Y, Shu J, Hardin J, et al. Peripubertal vitamin D3 deficiency delays puberty and disrupts the estrous cycle in adult female mice. Biol Reprod 2012;87:51.

8. Merhi Z, Doswell A, Krebs K, Cipolla M. Vitamin D alters genes involved in follicular development and steroidogenesis in human cumulus granulosa cells. J Clin Endocrinol Metab 2014;99:E1137-45.

9. Wojtusik J, Johnson PA. Vitamin D regulates anti-Mullerian hormone expression in granulosa cells of the hen. Biol Reprod 2012;86:91.

10. Durlinger AL, Visser JA, Themmen AP. Regulation of ovarian function: the role of anti-Müllerian hormone. Reproduction 2002;124:601-9.

11. Rotterdam ESHRE/ASRM-Sponsored PCOS Consensus Workshop Group. Revised 2003 consensus on diagnostic criteria and long-term health risks related to polycystic ovary syndrome. Fertil Steril 2004;81:19-25.

12. De Vos M, Devroey P, Fauser BC. Primary ovarian insufficiency. Lancet 2010;376:911-21.

13. Chandeying P, Pantasri T. Prevalence of conditions causing chronic anovulation and the proposed algorithm for anovulation evaluation. J Obstet Gynaecol Res 2015;41:1074-9.

14. Fauser BC, Tarlatzis BC, Rebar RW, Legro RS, Balen AH, Lobo $R$, et al. Consensus on women's health aspects of polycystic ovary syndrome (PCOS): the Amsterdam ESHRE/ASRM-Sponsored 3rd PCOS Consensus Workshop Group. Fertil Steril 2012;97:28-38.e25.

15. Holick MF, Binkley NC, Bischoff-Ferrari HA, Gordon CM, Hanley DA, Heaney RP, et al. Evaluation, treatment, and prevention of vitamin $\mathrm{D}$ deficiency: an Endocrine Society clinical practice guideline. J Clin Endocrinol Metab 2011;96:1911-30.

16. Wong HY, Li HW, Lam KS, Tam S, Shek CC, Lee CY, et al. Independent association of serum vitamin $D$ with anti-Mullerian hormone levels in women with polycystic ovary syndrome. Clin Endocrinol (Oxf) 2018;89:634-41.
17. Cappy H, Giacobini P, Pigny P, Bruyneel A, Leroy-Billiard $M$, Dewailly $D$, et al. Low vitamin D3 and high antiMüllerian hormone serum levels in the polycystic ovary syndrome (PCOS): is there a link? Ann Endocrinol (Paris) 2016;77:593-9.

18. Hahn S, Haselhorst U, Tan S, Quadbeck B, Schmidt M, Roesler $S$, et al. Low serum 25-hydroxyvitamin $D$ concentrations are associated with insulin resistance and obesity in women with polycystic ovary syndrome. Exp Clin Endocrinol Diabetes 2006;114:577-83.

19. Krul-Poel YH, Snackey C, Louwers Y, Lips P, Lambalk $C B$, Laven JS, et al. The role of vitamin D in metabolic disturbances in polycystic ovary syndrome: a systematic review. Eur J Endocrinol 2013;169:853-65.

20. Visser J. Role of anti-Müllerian hormone in follicle recruitment and maturation. J Gynecol Obstet Biol Reprod (Paris) 2006;35:2530-4.

21. Irani M, Merhi Z. Role of vitamin D in ovarian physiology and its implication in reproduction: a systematic review. Fertil Steril 2014;102:460-468.e3.

22. Merhi ZO, Seifer DB, Weedon J, Adeyemi O, Holman S, Anastos $\mathrm{K}$, et al. Circulating vitamin D correlates with serum antimüllerian hormone levels in late-reproductiveaged women: Women's Interagency HIV Study. Fertil Steril 2012;98:228-34.

23. Dennis NA, Houghton LA, Jones GT, van Rij AM, Morgan K, McLennan IS. The level of serum anti-Müllerian hormone correlates with vitamin D status in men and women but not in boys. J Clin Endocrinol Metab 2012;97:2450-5.

24. Jukic AM, Steiner AZ, Baird DD. Association between serum 25-hydroxyvitamin $D$ and ovarian reserve in premenopausal women. Menopause 2015;22:312-6.

25. Drakopoulos P, van de Vijver A, Schutyser V, Milatovic S, Anckaert $E$, Schiettecatte J, et al. The effect of serum vitamin $D$ levels on ovarian reserve markers: a prospective cross-sectional study. Hum Reprod 2017;32:208-14.

26. Irani M, Minkoff $H$, Seifer DB, Merhi Z. Vitamin D increases serum levels of the soluble receptor for advanced glycation end products in women with PCOS. J Clin Endocrinol Metab 2014;99:E886-90. 